\title{
RELAÇÃO ENTRE O PERFIL DO GESTOR E A PERCEPÇÃO DO AMBIENTE EXTERNO NA ELABORAÇÃO DA ESTRATÉGIA CORPORATIVA
}

\author{
Marino Luiz Eyerkaufer, Marines Lúcia Boff, Flávia de Souza \\ Universidade do Estado de Santa Catarina - UDESC \\ marino.luiz@udesc.br, marines.boff@udesc.br, flavia_grp@hotmail.com
}

\begin{abstract}
Resumo
A discussão do artigo contempla o ambiente externo às empresas e a relação do perfil do gestor na percepção deste na elaboração da estratégia corporativa. O ambiente externo que compreende entre outros elementos: o consumidor, a tecnologia, a economia e a política de uma sociedade, em tese deveriam ser pressuposto para a elaboração da estratégia corporativa. Nesta perspectiva, surge a discussão do estudo que de forma abrangente identifica a visão que os consumidores tem dos gestores no que diz respeito à relação da percepção do ambiente externo e o perfil estratégico na elaboração da estratégia corporativa nas empresas de Ibirama do Estado de Santa Catarina, Brasil. $\mathrm{O}$ arcabouço teórico permitiu a elucidação de vários aspectos relacionados com ambiente externo, perfil de gestor e estratégica corporativa bem como da satisfação dos consumidores. Trata-se de um estudo descritivo, que além da revisão bibliográfica, efetuou levantamento de dados in-loco pelo método de survey com consumidores no perímetro urbano da Cidade de Ibirama/SC em julho de 2013. Estudo semelhante foi efetuado onde os sujeitos foram os gestores das empresas, por isso, alguns dados comparativos nos permitem visualizar que há concordâncias e discordâncias na visão de gestores e consumidores quanto aos aspectos relacionados com o tema em estudo. Conclui-se que as características do perfil dos gestores e a percepção do ambiente externo apontam para lacunas no processo de elaboração da estratégia corporativa que satisfaça plenamente o consumidor.
\end{abstract}

Palavras-chave: Ambiente externo, Perfil estratégico, Estratégia corporativa.

\section{RELATION BETWEEN THE MANAGER'S PROFILE AND THE FEELING OF EXTERNAL ENVIRONMENT ON THE ELABORATION OF CORPORATE STRATEGY}

\begin{abstract}
The article includes discussion of the external environment to companies and the relation of the profile manager in the perception of the preparation of corporate strategy. The external environment which includes inter alia: consumer, technology, economics, and politics of a society, in theory should be a prerequisite for the development of corporate strategy. In this perspective, the discussion of the study that comprehensively identifies the view that consumers have of managers with regard to the relationship between the perception of the external environment and the strategic profile in the preparation of corporate strategy in business Ibirama the State of Santa Catarina arises, Brazil. The theoretical framework has allowed the elucidation of various aspects of the external environment, profile manager and strategic corporate as well as consumer satisfaction. This is a descriptive study, in addition to the literature review, data collection carried out in- situ by means of a survey with consumers within the city limits of the City of Morrow/SC in July 2013. A similar study was conducted in which subjects were managers of the companies, so some comparative data allow us to see that there are agreements and disagreements in the views of managers and consumers in the matters
\end{abstract}


related to the subject under study. We conclude that the characteristics of the profile of managers and perception of the external environment point to gaps in the process of formulating corporate strategy that fully meets the consumer.

Keywords : External Environment, Strategic Profile, Corporate Strategy.

\section{Introdução}

Com o aumento de concorrência aliado à maior exigência dos consumidores, emprego de estratégias corporativas arrojadas que conduzam efetivamente à vantagem competitiva é sem dúvida um caminho para manutenção ou ganho de market share das corporações.

Toda estratégia deveria surgir a partir do planejamento que se dá por um processo de definição da missão, de entender as necessidades que a empresa terá que satisfazer. A formulação da estratégia por sua vez, começa pelo conhecimento do seu negócio levando em conta aspectos internos (pontos fortes e fracos) e externos (oportunidades e ameaças).

Entende-se que a estratégia tem como objetivo satisfazer o consumidor, aquele que tem muitas opções na hora da escolha de um produto ou serviço. O comportamento do consumidor deve ser levado em conta na elaboração das estratégias corporativas. O processo de compra se inicia quando o consumidor tem uma necessidade a satisfazer.

Por outro lado, o perfil do gestor tem larga relação com a forma que este conduz seu negócio principalmente no que diz respeito à percepção que este tem do ambiente externo, dos quais um dos elementos é o consumidor.

$\mathrm{O}$ ambiente externo é o conjunto de elementos que exercem influencia sobre o ambiente interno das empresas, a exemplo do consumidor, a tecnologia, a economia e a política de uma sociedade (LACOMBE; HEILBORN, 2008).

De forma abrangente o artigo objetiva identificar a relação entre o perfil do gestor e a percepção do ambiente externo na elaboração da estratégia corporativa, a partir da pesquisa com os consumidores de Ibirama/SC. Ainda de forma específica buscou-se: identificar características dos gestores e a percepção do ambiente externo; identificar a satisfação do consumidor diante das estratégias corporativas adotadas pelas empresas; e por fim, a partir dos dados propor aos gestores estratégias capazes de elevar a satisfação dos consumidores.

O estudo descritivo utilizou-se de pesquisa bibliográfica e survey com análise quantitativa. Os dados foram coletados no perímetro urbano da cidade de Ibirama/SC em julho de 2013 em pontos de muita circulação de consumidores, com aplicação de questionário estruturado com perguntas fechadas. Dados do IBGE (2010) indicam que o Município possui 17.330 habitantes. A pesquisa atingiu 103 consumidores dos quais $92 \%$ são moradores de Ibirama/SC.

Sob o prisma teórico, o estudo tem sua importância destacada pela síntese de conceitos que relacionam a gestão, o ambiente externo e estratégia empresarial. A contribuição prática deste estudo oferece subsídios significativos para desenvolvimento dos gestores.

Neste trabalho inicialmente apresenta-se a introdução ao estudo, na segunda seção os subsídios teóricos. Na terceira seção a discussão dos dados da pesquisa, e por fim, na última seção as considerações do estudo.

\section{Subsídios teóricos}

Para maior elucidação do tema, aborda-se nesta seção uma breve fundamentação a cerca do ambiente organizacional, gestão e características do gestor e estratégia empresarial. 


\subsection{Ambiente organizacional}

A discussão primordial abrange o ambiente organizacional que pode ser entendido como tudo que envolve uma organização. Toda organização está inserida em uma rede de influências, interna e externa (PEREIRA, 2004).

A organização como sistema aberto está sujeita às influencias externas do ambiente. As empresas tem um objetivo de responder ao ambiente um propósito de rapidez e eficiência, sendo necessário conhecê-lo (PEREIRA, 2004).

O mapeamento do ambiente apresenta algumas dificuldades:

Seleção ambiental: Não é fácil conhecer todas as variáveis do ambiente, sendo assim é necessário escolher aqueles que têm um objetivo maior para a organização (PEREIRA, 2004).

Percepção Ambiental: refere-se a um conjunto de informação em função de experiências, ou seja, depende da expectativa, entendimento de cada organização (PEREIRA, 2004).

Limites ou fronteiras: é um entendimento da definição do que é ambiente e organização (PEREIRA, 2004).

O ambiente externo possui elementos de ação direta a exemplo dos stakeholders, já a ação indireta, é composta pela tecnologia, economia e a política de uma sociedade (LACOMBE; HEILBORN, 2008).

Os stakeholders podem ser entendidos em duas categorias, stakeholders externos que são compostos pelos sindicados, fornecedores, consumidores, e stakeholders internos que inclui os empregados, acionistas (LACOMBE; HEILBORN, 2008).

\subsection{Gestão e características do gestor}

Para Sobral e Peci (2008) as funções da administração são quatro: planejar, organizar, dirigir e controlar. O gestor deve considerar os efeitos relacionadas de cada uma (SOBRAL; PECI; 2008).

Primeiramente, no planejamento, onde os administradores têm em mente os objetivos da empresa, sabendo que eles podem ter o risco da incerteza. Organizar é a função de distribuir tarefas entre os membros da organização, alcançando uma melhor forma de seguir o que foi planejado. A função de direção está relacionada com liderar, motivar, coordenar os trabalhos desenvolvidos, proporcionando um lugar onde os trabalhadores se sintam realizados. A função do controle é aquela que atesta se os objetivos propostos no planejamento estão sendo alcançados (SOBRAL; PECI; 2008).

As habilidades esperadas de um gestor são conceituais, humanas e técnicas.

Habilidades Conceituais: As habilidades conceituais estão relacionadas com a capacidade do administrador coordenar e integrar todos os interesses e atividades de uma organização ou grupo. São as habilidades conceituais que permitem aos administradores analisar e interpretar situações abstratas e complexas e compreender como as partes influenciam o todo (SOBRAL; PECI; 2008).

Habilidades Humanas: As habilidades humanas dizem respeito à capacidade do administrador de se relacionar com outras pessoas ou grupos. Envolvem a capacidade de trabalhar e se comunicar com outras pessoas, entendendo-as, motivando-as e liderando-as. Consiste na realização dos objetivos por meio de outras pessoas, onde as habilidades humanas são cruciais para seu desempenho (SOBRAL; PECI; 2008).

Habilidades Técnicas: As habilidades técnicas estão relacionadas com a capacidade dos administradores usarem ferramentas, procedimentos, técnicas e conhecimentos especializados relativos à sua área de atuação específica. Quanto maior a facilidade em desempenhar uma tarefa específica, maiores as habilidades técnicas do administrador (SOBRAL; PECI; 2008). 
Quanto as características do gestor, a autoconfiança é uma delas, pois o empreendedor tem certeza do seu projeto e que há confiança na sua própria capacidade. Ele pode ser inovador, onde ele cria novos produtos, tem o dom de encontrar novas estratégias. O empreendedor pode ser caracterizado de várias formas, para ele ser um líder, ele tem que ter a capacidade de motivar e influenciar seus funcionários nas suas tarefas. Sendo automotivado, mesmo errando sempre começara tudo de novo. Para ser informado, precisa ser interassado em aprender os detalhes do seu negócio e o que está ao seu redor, mantendo-se sempre atualizado (DE OLIVEIRA; VIEIRA JUNIOR; DE OLIVEIRA; Et al, 2008).

Ainda se atribui como característica do gestor empreendedor a capacidade de assumir riscos, pois ao assumi-los é preciso ter a coragem de enfrentar os desafios, é buscar caminhos para um bom negócio. Porém, não basta ver a oportunidade do negocio sem agir de forma que este possa dar certo (FARAH; CAVALCANTI; MARCONDES, 2008).

A busca de informações é uma das características mais importantes, pois o gestor precisa conhecer o ramo que atua. O planejamento se torna essencial, pois ele cria um rumo a ser perseguido (FARAH; CAVALCANTI; MARCONDES, 2008).

\subsection{Administração e estratégia empresarial}

Administração é um sistema estruturado e intuitivo que consolida um conjunto de princípios, normas e funções para alcançar, harmoniosamente, o processo de planejamento de situações futuras desejadas e seu posterior controle de eficiência e produtividade, bem como a organização e direção dos recursos empresariais para os resultados esperados, com a minimização de conflitos interpessoais (DE OLIVEIRA, 1999, p. 26).

A estratégia identifica, analisa e efetiva a interligação entre fatores ambientais internos e externos, visando usufruir das oportunidades ou de minimizar as ameaças perante as fragilidades e fortalezas da organização (DE OLIVEIRA, 1999).

Corrobora Beal (2008) que a estratégia é o conjunto de decisões tomadas para a definição dos objetivos globais associados a um determinado período de tempo além da identificação dos meios mais adequados para superar os desafios e alcançar os objetivos.

A administração estratégica pode ser entendida pelos conceitos individualmente apresentados de administração e estratégia, que segundo De Oliveira (1999) representa uma administração que, de forma estruturada, sistêmica e intuitiva, consolida um conjunto de princípios, normas e funções para alavancar harmoniosamente o processo de planejamento do futuro esperado e posterior controle diante das mudanças ambientais, bem como organizar e dirigir os recursos de forma otimizada, maximizando as relações interpessoais.

Corrobora Bernardi (2003) que a administração estratégica é um modelo de administração flexível e dinâmico que se baseia na interação cooperativa e sistêmica; planejamento, organização e controle desenhados para a flexibilidade e iniciativa; autocrítica; inovação constante; abertura para mudanças e ênfase nestas antes dos resultados de curto prazo e valorização das características culturais com incentivo à iniciativa.

Peter e Certo (2005) por sua vez definem a administração estratégica como um processo contínuo e interativo que visa alinhar a organização com o ambiente.

Estratégia consiste em mudança que a empresa idealiza para atingir melhor desempenho, sendo entendida como um padrão que integra o negócio da empresa. O processo de planejar a estratégia começa com a definição do negócio, identificando a missão de qual necessidade do mercado que a empresa satisfaz (MAXIMIAMO, 2006).

Para (MILES; SNOW, 1978 apud BRESSAN Et. Al., 2011, p. 12), são quatro os tipos de estratégias: 
Estratégia Defensiva: uma empresa seguindo essa estratégia procura localizar e manter uma linha de produtos/ serviços relativamente estável. Possuem domínio estreito do produto e do mercado, mais limitado do que seus concorrentes e tentam proteger seu domínio através da oferta de produtos com melhor qualidade, serviços superiores e/ou menores preços. Não procura estar entre os líderes da indústria, restringindo-se aquilo que sabe fazer tão bem, ou melhor, que qualquer um. Não tendem a procura novas oportunidades fora do seu domínio.

Estratégia Prospectora: Procura novas oportunidades de negócios buscando ampliar continuamente sua linha de produtos/serviços; Valoriza ser uma das primeiras a oferecer novos produtos, mesmo que todos os esforços não se mostrem altamente lucrativos. Seus gestores são altamente flexíveis quando a mudança e a inovação;

Estratégia Analítica: Uma empresa que segue está estratégia tenta manter um alinha limitada de produtos/serviços relativamente estável e ao mesmo tempo tenta adicionar um ou mais novos produtos/serviços que foram bem sucedidos em outras empresas do setor. Protegem a porção estável do seu mercado de atuação;

Estratégia Reativa: Não possui habilidade para responder efetivamente as implicações das mudanças ocorridas no seu ambiente; Não arriscam em novos produtos/ serviços a não ser quando ameaça por competidores; É uma espécie de não estratégia. A abordagem típica é esperar para ver e responder somente quando forçada por pressões competitivas para evitar a perda de clientes importantes e/ou manterem lucratividade. Esses tipos de estratégias tem uma diferença nas escolhas de como as pessoas utilizam as informações descritas em cada tipo de estratégias, sendo assim o empreendedor terá uma visão ampla de como há uma formulação estratégica.

Para a formulação das estratégias, é necessário o empreendedor ter em mente algumas perguntas e respostas, referente ao seu negócio (CERTO; PETER; 2005). Primeiramente, ele precisa saber quais são os objetivos e propósitos da organização e quais os fatores críticos que a organização enfrenta. Depois ele precisa analisar os fatores externos e internos que envolvem os riscos da empresa, apresentando os pontos fracos e fortes (CERTO; PETER; 2005).

As estratégias tem uma formulação organizacional, porém a sua administração faz parte da sua formação, projetando os objetivos das empresas (CERTO; PETER; 2005). Já o controle estratégico se concentra no monitoramento e avaliação do processo de administração estratégico, para poder alcançar todos os resultados do planejamento (CERTO; PETER; 2005).

Após conceituar estratégia, entende-se que esta será empregada para alcançar o mercado, entendido atualmente em constante mutação permitindo maiores dificuldades de penetração bem como de manutenção do market share.

O mercado é um conjunto de pessoas que tem poder de compra. De acordo com Maximiliano (2006, p.88) o "mercado é um grupo de consumidores que tem a necessidade e interesse similares, poder aquisitivo e disposição para compra".

Ainda se define como uma população total, ou seja, se refere ao segmento economicamente ativo, que utiliza os produtos das empresas. Há dois tipos de mercados: mercado consumidor e mercado industrial. O mercado consumidor compra o produto para si mesmo, ou seja, para o seu próprio uso. Já o mercado industrial, compra os produtos para serem revendidos (MAXIMIAMO, 2006). A segmentação do mercado é a divisão dos consumidores, tendo um objetivo em grupo, dessa forma as organizações podem desenvolver uma oferta mais adequada aos segmentos (SOBRAL e PECI, 2008).

Sobral e Peci (2008, p. 307) afirmam que a organização deve:

a) Compreender os benefícios que os consumidores esperam;

b) Dividir o mercado de acordo com as características dos clientes para compor cada segmento;

c) Descobrir as variáveis observáveis que melhor discriminem os segmentos;

O produto é considerado um elemento importante da estratégia, sendo assim o mercado deve produzir para cada público que considera seu alvo (SOBRAL e PECI, 2008). 
A satisfação dos consumidores é referente a um produto após a sua compra, essa satisfação só se dá quando o resultado das suas necessidades será alcançado pela a correspondência das características dos produtos (MOWEN; MINOR, 2003).

Outro fator relevante para elaboração da estratégia corporativa é a definição do público que se pretende atingir, mormente dividido em classes sociais definidos pelo fator chamado renda, que se torna um importante indicador de consumo, pois revela o poder aquisitivo de cada classe (SILVA; BARBOS, 2011). Demonstra-se na Tabela 01 as classes sociais de acordo com a renda.

\begin{tabular}{c|c|c}
\hline Classe Social & Renda Mensal ( $n^{\circ}$ de salários mínimos) & Renda Mensal em $R \$$ \\
\hline A & Mais de 15 & Mais de R\$ 10170,00 \\
\hline B & De 05 a 15 & De R $\$ 3390,00$ a R\$ 10170,00 \\
\hline C & De 03 a 05 & De R \$ 2034,00 a R $\$ 3390,00$ \\
\hline D & De 01 a 03 & De R $\$ 678,00$ a R $\$ 2034,00$ \\
\hline E & Até 01 & Até R $\$ 678,00$ \\
\hline
\end{tabular}

Tabela 01- Classes Sociais de acordo com a renda ( ADAPTADO DE TEXEIRA, 2009; PNAD, 2008; ABEP, 2009

Para efeitos de cálculo considerou-se o valor do salário mínimo de $\mathrm{R} \$ 678,00$ que vigorou no ano de 2013.

\section{Dados do estudo}

Apresenta-se e se discute os dados coletados, onde inicialmente buscou-se identificar o vinculo com a cidade de cada sujeito entrevistado.

\begin{tabular}{l|c|c}
\hline \multicolumn{1}{c|}{ Vinculo com a cidade de Ibirama/SC } & $N^{\circ}$ & $\%$ \\
\hline Reside e trabalha em Ibirama/SC & 90 & $87 \%$ \\
\hline Reside em Ibirama/SC e trabalha em outro município & 5 & $5 \%$ \\
\hline Trabalha em Ibirama/SC e reside em outro município & 8 & $8 \%$ \\
\hline Total & 103 & $100 \%$ \\
\hline
\end{tabular}

$\overline{\text { Tabela 01- Vínculo os entrevistados com a cidade de Ibirama/SC (Dados da pesquisa, 2013) }}$

Nota-se que a maioria dos entrevistados reside e trabalha em Ibirama/SC, porém, uma pequena parte dos entrevistados trabalha em Ibirama e reside em outros municípios da região.

Os dados revelam também que $46 \%$ dos entrevistados são do sexo masculino e $51 \%$ feminino. Ainda que $6 \%$ jovens com idade até 20 anos, 32\% tem idade de 21 a 30 anos, 31\% com uma idade de 41 a 50 anos, $10 \%$ possuem idade de 51 a 60 anos, e 5\% considerado um público mais idoso, com idade acima de 61 anos.

Quanto a escolaridade, os dados revelam que $31 \%$ dos consumidores possui o segundo grau completo, e significativo número cursou ou está cursando ensino superior.

Apresenta-se na Tabela 02 a classe de consumo dos entrevistados da cidade de Ibirama/SC.

\begin{tabular}{l|c|c}
\hline \multicolumn{1}{c|}{ Classe de consumo de acordo com a renda familiar } & $N^{o}$ & $\%$ \\
\hline Classe A - Renda superior a $\mathrm{R} \$ 10.170,00$. & 1 & $1 \%$ \\
\hline Classe B - Renda superior de $\mathrm{R} \$ 3.390,00$ a $10.170,00$ & 16 & $16 \%$ \\
\hline Classe C - Renda superior de $\mathrm{R} \$ 2.034,00$ a $3.390,00$ & 22 & $21 \%$ \\
\hline
\end{tabular}




\begin{tabular}{l|c|c} 
Classe D - Renda superior de R\$ 678,00 a $2.034,00$. & 49 & $51 \%$ \\
\hline Classe E - Renda até R\$ 678,00 & 15 & $15 \%$ \\
\hline Total & 103 & $100 \%$ \\
\hline
\end{tabular}

Tabela 02 - Classe de consumo dos consumidores entrevistados da cidade de Ibirama/SC (ADAPTADO DE TEXEIRA, 2009; PNAD, 2008; ABEP, 2009)

Percebe-se que a maioria dos entrevistados é da classe D, porém a minoria apresenta classe A. Para elaboração das estratégias empresariais, entende-se que é de suma importância o gestor levar em conta o poder aquisitivo dos consumidores.

Apresenta-se na Tabela 03 a opinião dos consumidores em relação ao emprego das funções da administração pelos gestores de Ibirama/SC.

\begin{tabular}{l|c|c}
\hline $\begin{array}{l}\text { Emprego das funções da administração: planejamento, controle, } \\
\text { coordenação e direção. }\end{array}$ & $N^{\circ}$ & $\%$ \\
\hline Muito & 3 & $3 \%$ \\
\hline Nem muito e nem pouco & 90 & $87 \%$ \\
\hline Pouco & 10 & $10 \%$ \\
\hline Total & 103 & $100 \%$ \\
\hline
\end{tabular}

Tabela 03 - Funções da administração e seu emprego pelos gestores na opinião dos entrevistados (DADOS DA PESQUISA, 2013)

Percebe-se que para a maioria dos entrevistados as funções da administração que são o "planejamento, controle, coordenação e direção", são nem muito e nem pouco empregados pelos gestores, o que revela que na opinião dos consumidores há fragilidades na gestão das empresas.

$\mathrm{Na}$ Tabela 04 apresenta-se as habilidades mais marcantes que os consumidores de Ibirama/SC percebem nos gestores.

\begin{tabular}{l|c|c}
\hline $\begin{array}{l}\text { Habilidades mais marcantes que os consumidores percebem nos } \\
\text { gestores de Ibirama/SC }\end{array}$ & $N^{\circ}$ & $\%$ \\
\hline Técnica & 85 & $83 \%$ \\
\hline Humana/gerencial & 14 & $14 \%$ \\
\hline Conceitual & 4 & $4 \%$ \\
\hline Total & 103 & $100 \%$ \\
\hline
\end{tabular}

Tabela 04 - Habilidades mais marcantes em que os consumidores percebem nos gestores da cidade de Ibirama/SC (DADAS DA PESQUISA, 2013)

Mostra-se na Tabela 04 que a maioria dos consumidores entrevistados, nota que a habilidade mais marcante nos gestores é a técnica. A habilidade técnica relaciona-se com a atividade específica do gestor, os conhecimentos, métodos e equipamentos necessários para a realização das tarefas que dizem respeito ao negócio. A habilidade humana ou gerencial abrange a capacidade de entender, liderar e trabalhar com pessoas. Já a habilidade conceitual envolve a capacidade de compreender e liderar a complexidade de toda a organização e de usar o intelecto para formular estratégicas, criatividade, planejamento, raciocínio abstrato e entendimento do contexto (SOBRAL; PECI, 2008).

Apresenta-se na Tabela 05 as estratégias que os gestores de Ibirama/SC adotam na opinião dos consumidores.

\begin{tabular}{|c|c|c|}
\hline Em relação às estratégias, qual que os gestores de Ibirama/SC mais utilizam: & $N^{o}$ & $\%$ \\
\hline Produtos/serviços estáveis & 44 & $45 \%$ \\
\hline Produtos/serviços em constante inovação & 13 & $13 \%$ \\
\hline
\end{tabular}


Inova pouco em produtos/serviços apenas compra o que já deu certo na concorrência

Não arriscam em novos produtos/serviços a não ser quando ameaçados por competidores

Não sabe opinar

Total

Tabela 05- Relação às estratégias, em que os entrevistados da cidade de Ibirama/SC acreditam que os gestores mais utilizam (DADOS DA PESQUISA, 2013)

Nota-se que $45 \%$ dos entrevistados acredita que a estratégia preponderante dos gestores de Ibirama é de produtos/serviços estáveis seguido de $29 \%$ que não arriscam em novos produtos/serviços a não ser quando ameaçados por competidores.

De acordo com (MILES; SNOW, 1978 apud BRESSAN, TOLEDO, DUGAHARA ET. AL., 2011), trata-se da estratégia defensiva e reativa onde a primeira se caracteriza por manter uma linha de produtos/serviços relativamente estável e a segunda por não apresentar habilidades para responder efetivamente as implicações das mudanças do ambiente.

Indagados sobre outras oportunidades de resposta à questionários de satisfação, $58 \%$ dos consumidores responderam que já haviam respondido a outros questionários.

Apresenta-se na Tabela 06 a visão dos consumidores quanto as estratégias corporativas, o que diz respeito a observância dos gostos dos consumidores na sua elaboração.

\begin{tabular}{l|c|c}
\hline Seus gostos e preferências são importantes para os gestores & $\mathbf{N}^{\mathbf{0}}$ & $\mathbf{\%}$ \\
\hline Sempre & 4 & $4 \%$ \\
\hline Às vezes & 74 & $72 \%$ \\
\hline Pouco & 10 & $10 \%$ \\
\hline Nunca & 15 & $15 \%$ \\
\hline Total & 103 & $100 \%$ \\
\hline
\end{tabular}

Tabela 06 - Os gostos e preferências dos consumidores entrevistados da cidade de Ibirama/SC e sua observância pelos empresários na hora do planejamento (DADOS DA PESQUISA, 2013)

Nota-se que os gostos dos consumidores nem sempre são importantes para os empresários na hora do planejamento das estratégias.

Apresenta-se na Tabela 07 o porcentual das compras realizadas do comércio de Ibirama/SC.

\begin{tabular}{l|c|r}
\hline Porcentual de compras realizada no comércio de Ibirama/SC & $\mathbf{N}^{\mathbf{0}}$ & $\mathbf{\%}$ \\
\hline $100 \%-80 \%$ & 64 & $62 \%$ \\
\hline $79 \%-60 \%$ & 16 & $16 \%$ \\
\hline $59 \%-40 \%$ & 5 & $5 \%$ \\
\hline Abaixo de 39\% & 18 & $17 \%$ \\
\hline Total & 103 & $100 \%$ \\
\hline
\end{tabular}

Tabela 07- Porcentual de compras realizadas do comércio da cidade de Ibirama/SC. (DADOS DA PESQUISA, 2013)

Nota-se que apenas $62 \%$ dos consumidores consomem acima de $80 \%$ no comércio de Ibirama/SC. Levando em conta que $92 \%$ dos entrevistados residem em Ibirama, o índice de consumidores que efetuam menos de $39 \%$ das suas compras em Ibirama é significativo.

$\mathrm{Na}$ Tabela 08 apresenta-se os motivos que motivam a compra dos consumidores de Ibirama/SC.

Por qual motivo costuma comprar

Oferta de determinado produtos

Pela sua necessidade

\begin{tabular}{|c|c|}
\hline $\mathbf{N}^{\mathbf{0}}$ & $\mathbf{\%}$ \\
\hline 45 & $44 \%$ \\
\hline 50 & $49 \%$ \\
\hline
\end{tabular}


Pelas sensações, sentimentos, imagens, emoção que os produtos geram

Outros. Especifique

Total

\begin{tabular}{c|c}
8 & $8 \%$ \\
\hline 0 & $0 \%$ \\
\hline 103 & $100 \%$ \\
\hline
\end{tabular}

Tabela 08- Motivos pelo qual os consumidores compram na cidade de Ibirama/SC. (DADOS DA PESQUISA, 2013).

Nota-se que $49 \%$ dos consumidores compram pela sua necessidade de compra, $44 \%$ compram pela a oferta dos produtos, e $8 \%$ compram pela as suas sensações, sentimentos, imagens, emoção em que os produtos geram. O resultado demonstra que a compra por impulso não é fator determinante na hora da compra.

Apresenta-se na Tabela 09 o que os entrevistados gostariam que melhorasse para a sua satisfação.

O que gostaria que as empresas de Ibirama/SC melhorassem para a sua satisfação

Estou $100 \%$ satisfeito

Diminuir os preços

Melhorar o atendimento

Diversificar mais produtos

Total

\begin{tabular}{|c|c|}
$N^{o}$ & $\%$ \\
\hline 3 & $3 \%$ \\
\hline 40 & $39 \%$ \\
\hline 18 & $41 \%$ \\
\hline 103 & $17 \%$ \\
\hline
\end{tabular}

Tabela 09- Motivos para o melhoramento da satisfação dos consumidores da cidade de Ibirama/SC. (DADOS DA PESQUISA, 2013).

Nota-se que $41 \%$ dos consumidores entrevistados gostariam que o atendimento no comércio de Ibirama/SC melhorasse, 39\% gostariam que os preços diminuíssem, 17\% gostariam que os produtos fossem diversificados e 3\% estão cem por cento satisfeito.

Apresenta-se na Tabela 10 os elementos que os consumidores consideram suficientes ou insuficientes na hora da compra em Ibirama/SC.

\begin{tabular}{l|c|c|c|c}
\hline Na hora de comprar em Ibirama/SC & suficiente & $\%$ & Insuficiente & $\%$ \\
\hline Produtos e serviços estão dispostos em todas as informações & 50 & $49 \%$ & 53 & $51 \%$ \\
\hline Respeitam minha lealdade a marca & 51 & $50 \%$ & 52 & $50 \%$ \\
\hline Permitem o tempo que eu preciso para decidir & 56 & $54 \%$ & 47 & $46 \%$ \\
\hline
\end{tabular}

Tabela 10- Elementos que os consumidores acham suficientes e/ou insuficientes na hora da compra em Ibirama/SC. (DADOS DA PESQUISA, 2013).

Nota-se que praticamente a metade dos consumidores acha suficiente a disposição dos produtos e serviços e com todas as informações, que as empresas respeitam a lealdade a marca e ainda que permitem o tempo que precisam para decidir.

Ainda atendendo ao objetivo de estudo, apresenta-se no Quadro1, um paralelo de respostas que revela a opinião da maioria quanto as questões levantadas no estudo com gestores e mais tarde consumidores de Ibirama/SC.

\begin{tabular}{|l|lr|}
\hline \multicolumn{1}{|c|}{ NA OPINIÃO DOS GESTORES } & \multicolumn{3}{|c|}{ NA OPINIÃO DOS CONSUMIDORES } \\
\hline $\begin{array}{l}\text { Tem dificuldade de identificar a origem dos seus } \\
\text { clientes e sua classe de consumo além de revelar que } \\
\text { adotam novas estratégias sempre que acham } \\
\text { convenientes. }\end{array}$ & $\begin{array}{l}\text { Seus gostos e preferências apenas as vezes são } \\
\text { importantes para os gestores. }\end{array}$ \\
\hline $\begin{array}{l}\text { Em relação às estratégias adotadas na sua gestão, a } \\
\text { alternativa que melhor se enquadra com o gestor, é a } \\
\text { procura de novas oportunidades de negócio, buscando }\end{array}$ & $\begin{array}{l}\text { Em relação as estratégias empresariais, na opinião dos } \\
\text { consumidores, a predominante é a de } \\
\text { produtos/serviços estáveis. }\end{array}$ \\
$\begin{array}{l}\text { ampliar continuamente a sua linha de } \\
\text { produtos/serviços. ESTRATÉGIA PROSPECTORA. }\end{array}$ & $\begin{array}{l}\text { DEFENSIVA. } \\
\text { Características mais predominantes no seu perfil são } \\
\text { comunicação, negociação, criatividade e inovação. }\end{array}$ & $\begin{array}{l}\text { Característica em que mais percebe nos gestores são a } \\
\text { negociação e comunicação. }\end{array}$ \\
\hline
\end{tabular}




\begin{tabular}{|l|l|}
\hline $\begin{array}{l}\text { Das funções da administração, a que mais utiliza com } \\
\text { frequência em sua gestão é o planejamento. }\end{array}$ & $\begin{array}{l}\text { Das funções da administração, a utilização no } \\
\text { conjunto ou isoladamente pelos os gestores é nem } \\
\text { muito e nem pouco. }\end{array}$ \\
\hline $\begin{array}{l}\text { Das habilidades do gestor a mais destacada é a } \\
\text { técnica. }\end{array}$ & $\begin{array}{l}\text { Das habilidades do gestor, a mais destacada pelos } \\
\text { consumidores também é a técnica. }\end{array}$ \\
\hline
\end{tabular}

Quadro 01 - Opinião dos gestores e dos consumidores, relacionados a percepção do ambiente externo, características do gestor e gestão estratégica (DADOS DA PESQUISA, 2013)

É notável a concordância em vários aspectos pesquisados na opinião dos gestores das empresas e dos seus consumidores, outros revelam discordância. Mais uma vez se reforça a importância do gestor observar e perceber o ambiente externo, onde o consumidor como "eterno" insatisfeito dá sinais para o desenvolvimento de estratégias que realmente elevem a sua satisfação.

A partir da análise dos dados, atendendo ao objetivo do estudo, sugerem-se aos gestores algumas estratégias que possam elevar a satisfação dos consumidores de Ibirama/SC:

- Na elaboração das estratégias levar em conta os gostos e preferências do consumidor uma vez que os dados revelam que apenas as vezes isso ocorre.

- A maioria dos consumidores compra por uma necessidade, ou seja, só adquirem o produto quando necessário, outros compram pela oferta dos preços, aproveitam que tal produto está na promoção para adquirir, assim economizará mais, e a minoria consome por sensações ou, sentimentos. Nota-se que o publico consome pela sua necessidade e pela oferta dos produtos e apenas a minoria compra por impulso. Esta tendência precisa ser levada em conta pelos gestores.

\section{Considerações finais}

Objetivou-se neste estudo de forma abrangente identificar a relação do perfil do gestor e a percepção do ambiente externo na elaboração da estratégia corporativa através de um ensaio a partir da pesquisa com consumidores de Ibirama/SC.

$\mathrm{O}$ perfil dos consumidores se resume pelo equilíbrio entre os gêneros, com idade predominante de 21 a 40 anos e ainda a predominância da classe D de consumo, sendo 92\% moradores da cidade de Ibirama/SC.

Para os consumidores a estratégia de gestão é de produtos/serviços estáveis, ou seja, não veem como ousada a atuação dos empresários de Ibirama/SC. Ainda revelam que os gestores não utilizam as funções da administração plenamente e que seus gostos e preferências nem sempre são levados em conta na elaboração da estratégia empresarial.

Alguns dados revelam que o percentual de compras realizadas na cidade é relativamente baixo, ainda que apenas 3\% se dizem totalmente satisfeitos com as empresas das quais compra, e ainda que a disposição bem como de maiores informações para os produtos e serviços poderia ser melhorada.

Dentre as proposições de estratégias empresariais a partir da investigação com os consumidores, sugere-se de forma geral a observância dos gostos e preferências do consumidor na elaboração das mesmas bem como da melhoria do atendimento somado a estratégias de promoção por preço e produtos desejados uma vez que a minoria adquire por impulso.

Por fim, atendendo ao objetivo geral do estudo, conclui-se que as características do perfil dos gestores e a percepção do ambiente externo apontam para lacunas no processo de elaboração da estratégia corporativa que satisfaça plenamente o consumidor. 


\section{Referências}

BEAL, Adriana. Gestão estratégica da informação. São Paulo: Atlas, 2008.

BERNARDI, Luiz Antonio. Manual de empreendimento e gestão: fundamentos, estratégias e dinâmicas. São Paulo: Atlas, 2003.

BRESSAN, Flávio; TOLEDO,Geraldo Luciano; SUGAHARA, Cibele Roberta. Modelos mentais e as escolhas estratégicas de um empreendedor: tipologia de Miles e Snow sob a ótica do modelo de temperamentos de Keirsey e Bates. 2011. Disponível em:http://www.ead.fea.usp.br/semead/14semead/resultado/trabalhosPDF/1078.pdf. Acesso em: $26 / 02 / 2013$

DE OLIVEIRA, Djalma de Pinho Rebouças. Excelência na administração estratégica. 4. Ed. São Paulo: Atlas, 1999.

DE OLIVEIRA, Gian Márcio Paiva; VIEIRA JUNIOR, Eufrásio ; DE OLIVEIRA, Janyslandia Dias. O Perfil do empreendedor dos empresários do Brejo Paraibano. 2008. Disponível em: http://www.prac.ufpb.br/anais/xenex xienid/x enex/ANAIS/Area7/7CFTDCBSPEX01.pdf.

Aceso em:26/02/2013.

FARAH, Osvaldo Elias; CAVALCANTI, Marly,; MARCONDES, Luciana Passos. Empreendedorismo estratégico: criação e gestão de pequenas empresas. São Paulo: Cengage Learning, 2008.

IBGE - Instituto Brasileiro de Geografia e Estatística. Censo demográfico 2010. Disponível em: http://censo2010.ibge.gov.br. Acesso em: 28/01/2014.

LACOMBE, Francisco José Masset; HEILBORN, Gilberto Luiz José. Administração: princípios e tendências. 2.ed. rev. e atual. São Paulo: Saraiva, 2008.

MAXIMIANO, Antônio Cesar Armau. Administração para empreendedores: fundamentos da criação e da gestão de novos negócios. São Paulo: Pearson Hall, 2006.212 p.

MOWEN, John C; MINOR, Michael. Comportamento do consumidor. São Paulo: Prentice Hall, 2003.

PEREIRA, Ana Maris. Introdução à administração. 3. ed. São Paulo: Prentice Hall, 2004.

PETER, J. Paul; CERTO, Samuel C. Administração estratégica: planejamento e implantação da estratégia. 2. ed. São Paulo: Makron Books, 2005.

SOBRAL, Filipe; PECI, Alketa. Administração: teoria e prática no contexto brasileiro. São Paulo: Pearson Prentice Hall, 2008. 